\title{
Deaths in risk classes I-III: a measure of quality of care in patients hospitalised with CAP?
}

\author{
T.J. Marrie
}

Deaths in risk classes I-III: a measure of quality of care in patients hospitalised with CAP? T.J. Marrie. (C) ERS Journals Ltd 2004.

ABSTRACT: All deaths occurring in patients with community-acquired pneumonia in risk classes I-III were reviewed as a quality-of-care measure. The immediate and underlying causes of death were classified according to the World Health Organization protocol. Eleven $(1.8 \%)$ of the 608 low-risk patients died. Three of the patients did not have pneumonia, one of whom was admitted with atypical pulmonary oedema due to stenosis of a prosthetic heart valve. Failure to include chronic lung disease in the severity-of-illness scoring system resulted in misclassification of seven patients.

The most common underlying causes of death were pulmonary fibrosis at $27 \%$, chronic obstructive lung disease at $18 \%$ and cancer at $27 \%$. Respiratory failure was the immediate cause of death in $64 \%$ of patients, cardiac causes in $27 \%$, and pneumonia in $9 \%$.

To conclude, the review of deaths in patients at low risk for mortality is useful for monitoring the quality of care received by patients who require admission for the treatment of community-acquired pneumonia, and that the pneumonia-specific severityof-illness scoring system results in misclassification of patients with chronic obstructive lung disease and pulmonary fibrosis.

Eur Respir J 2004; 23: 103-105.
Correspondence: T.J. Marrie, 2F1.30 Walter C. Mackenzie Health Sciences Center, $8440-$ 112 St, Edmonton, AB, T6G 2B7, Canada. Fax: 17804073132

E-mail: tom.marrie@ualberta.ca

Keywords: Low risk

mortality

pneumonia

quality of care

Received: June 252003

Accepted after revision: August 162003

This work was supported by grants-in-aid from Capital Health Authority (Edmonton, AB), Pfizer Canada, Jannsen Ortho Canada, and Abbot Canada (Montreal, QC), and also by an establishment grant from Alberta Heritage Foundation for Medical Research, Edmonton, AB, Canada.
Community-acquired pneumonia (CAP) is a common illness with significant morbidity and mortality. Approximately 600,000 persons are hospitalised with pneumonia each year and there are 64 million days of restricted activity due to this illness $[1,2]$. Studies of CAP that exclude immunosuppressed persons with this illness report a mortality rate of $\sim 8 \%$ [3, 4]. FINE et al. [4] assigned points to 20 different factors, known to adversely affect the outcome from pneumonia, to derive a pneumonia-specific severity-of-illness (PSI) score. This system allowed categorisation of patients with pneumonia into five strata (risk classes I-V) with increasing risk for mortality. Patients in risk classes I-III who required admission to hospital for treatment of pneumonia had mortality rates of $0.5 \%, 0.9 \%$ and $1.25 \%$, respectively [4].

A pathway for the treatment of CAP was recently introduced in the city of Edmonton wherein all patients who present to the emergency room and have a diagnosis of pneumonia made are followed. It was hypothesised that a review of deaths among patients with CAP in risks groups I-III would serve as a quality-of-care measure.

\section{Methods}

\section{Study sites}

This study involved all six hospitals in the Edmonton area: two tertiary care hospitals, two hospitals that provided secondary and some tertiary care, and two community hospitals. This study was approved by the Research Ethics Committee at the University of Alberta and for use at all six study sites. The population of the city of Edmonton and its surrounding municipalities is 921,000 people [5].

\section{Development of pneumonia pathway}

A multidisciplinary team consisting of internists, physicians, respirologists, emergentologists, family physicians, pharmacists, nurses, respiratory therapists, and dietitians developed a comprehensive pathway for the management of CAP. The pathway consisted of guidelines for admission, antibiotic therapy and discharge [6]. The PSI score developed by the pneumonia Patient Outcomes Research Team (PORT) [4] was used to guide the admission decision. Six research nurses were hired to assist with implementing the pathway, perform data collection, and carry out follow-up phone calls 48-72 h following emergency room visits. Implementation began on November 15, 2000.

\section{Study population}

Patients were enrolled into the pathway if they presented to the emergency department for adults at one of the six hospitals in the Edmonton area with two or more symptoms or signs of CAP, plus radiographical evidence of pneumonia as interpreted by the emergency room physician or internal medicine consultant. Symptoms and signs of CAP included cough (productive or nonproductive), pleuritic chest pain, shortness of breath, temperature $>38^{\circ} \mathrm{C}$, and crackles on auscultation. Patients were excluded from the pathway if they 
had tuberculosis or cystic fibrosis. Pregnant and nursing mothers and immunosuppressed patients $(>10 \mathrm{mg}$ prednisone per day or other immunosuppressive drugs) were also excluded.

\section{Deaths in risk classes I-III}

In-hospital deaths in patients classified as risk classes I-III were recorded by study nurses, and the medical records and chest radiographs were re-reviewed by the author. The underlying and immediate causes of death were assigned based on World Health Organization (WHO) criteria [7]. The underlying cause of death was defined as the disease or injury that initiated the cause of morbid events leading directly to death. The immediate cause of death was defined as the disease process or injury or complication immediately preceding death $[7,8]$.

\section{Results}

During the first year of the pathway, 1,577 patients were admitted to the participating hospitals with pneumonia. Of these, $123(7.8 \%)$ died. No deaths occurred among the 28 patients in risk class I. There were three deaths among the 288 ( $1 \%$ ) deaths in risk class II, and eight among the $292(2.7 \%)$ patients in risk class III. Thus, 11 of $608(1.8 \%)$ patients in risk classes I-III died. Table 1 gives the salient features of the study population. There are two striking findings: three patients did not have pneumonia and in a fourth with interstitial lung disease it was impossible to determine whether pneumonia was or was not present; two had known malignancies and a third had carcinomatosis found at autopsy. The only preventable death occurred in the first patient in table 1. This 40-yr-old female had an aortic valve replacment 9 yrs previously because of aortic stenosis. The patient delivered a baby 8 weeks prior to presentation with cough and shortness of breath. The admission chest radiograph showed a right lower lobe opacity that was interpreted by the radiologist as probable pneumonia. The patient was afebrile. An echocardiogram showed a gradient of $70 \mathrm{mmHg}$ across the aortic prosthesis. On hospital day 3, the patient collapsed while walking to the bathroom and could not be resuscitated. An autopsy showed aortic stenosis and no evidence of pneumonia.
In retrospect, the admission chest radiograph was reinterpreted as atypical pulmonary oedema.

Table 1 also gives the underlying and immediate causes of death. Pneumonia was the immediate cause of death in one patient.

\section{Discussion}

The quality of medical care is usually measured by assessing the performance of the processes of care and the outcomes of these processes. Processes of care that are commonly assessed to judge the quality of care delivered to patients with pneumonia include performance of blood cultures within $24 \mathrm{~h}$ and prior to administration of antibiotics, outpatient care when appropriate, administration of antibiotics within $8 \mathrm{~h}$ of arrival at the hospital, oxygenation assessment, antibiotic therapy in accordance with published guidelines, switch from intravenous to oral antibiotics, length of stay, assessment of pneumococcal and influenza vaccination status and administration of these vaccines as appropriate, and smoking cessation counselling for those who smoke tobacco [9]. Mortality is the outcome measure for some of these processes of care.

The mortality rate in CAP varies considerably according to severity of illness [4]. The PSI developed by FINE et al. [4] stratifies patients into five risk classes with mortality ranging from $0.1 \%$ in risk class I to $26.7 \%$ in risk class $V$. In a recent administrative database study of all patients admitted to Alberta hospitals with pneumonia from April 1994 to March 1995 , it was found that the overall in-hospital mortality rate for these 43,642 patients was $11 \%$ [10]. Mortality was associated with severity of illness and comorbidity. In addition, it was found that physician experience was associated with mortality, in that physicians who cared for $>27$ patients with pneumonia per year had a lower in-hospital mortality rate [10].

A review of in-hospital deaths is a common practice in many hospitals as a means of ensuring quality care. Reviews of all deaths of patients with pneumonia are often impractical and of low yield in terms of quality improvement issues. Thus, a review of patients who died in risk classes I-III was felt to be a useful and practical measure of quality of care. It is useful given that this is a group that should be at low risk for

Table 1.-Selected features, underlying and immediate cause of death for 11 patients admitted for treatment of communityacquired pneumonia

\begin{tabular}{|c|c|c|c|c|c|c|c|}
\hline $\begin{array}{l}\text { Age } \\
\text { yrs }\end{array}$ & Sex & Comorbidities & Pneumonia & $\begin{array}{c}\text { Day of } \\
\text { death }\end{array}$ & $\begin{array}{l}\text { Underlying cause } \\
\text { of death }\end{array}$ & $\begin{array}{l}\text { Score, risk } \\
\quad \text { class }\end{array}$ & $\begin{array}{l}\text { Immediate cause } \\
\text { of death }\end{array}$ \\
\hline 40 & $\mathrm{~F}$ & Prosthetic aortic valve & No & 3 & Aortic stenosis & 60, II & Cardiac arrest \\
\hline 70 & $\mathrm{~F}$ & End stage COPD & Yes, Streptococcus pneumoniae & 7 & COPD & 90, III & Respiratory failure \\
\hline 53 & M & Cirrhosis & Yes, aspiration & 23 & Alcoholism & 73 , III & Alcohol withdrawal \\
\hline 63 & $\mathrm{~F}$ & $\begin{array}{l}\text { Lymphangitic spread of } \\
\text { breast cancer }\end{array}$ & Yes & 6 & Cancer & 84 , III & Respiratory failure \\
\hline 75 & $\mathrm{~F}$ & End stage COPD & No & 21 & COPD & 85 , III & Respiratory failure \\
\hline 34 & $\mathrm{~F}$ & $\begin{array}{l}\text { End stage pulmonary } \\
\text { fibrosis }\end{array}$ & $\begin{array}{l}\text { Impossible to determine, diffuse } \\
\text { pulmonary infiltrates in setting } \\
\text { of known pulmonary fibrosis }\end{array}$ & 13 & Pulmonary fibrosis & $54, \mathrm{II}$ & Respiratory failure \\
\hline 60 & $\mathrm{~F}$ & $\begin{array}{l}\text { Interstitial pneumonitis, } \\
\text { RA }\end{array}$ & No & 18 & Pulmonary fibrosis & 81 , III & Respiratory failure \\
\hline 71 & M & COPD, MI & Yes & 10 & Pneumonia & 71 , III & MI \\
\hline 41 & $\mathrm{~F}$ & Malignancy & Yes, aspiration & 10 & Cancer & 60, II & Pneumonia \\
\hline 69 & M & Pulmonary fibrosis & Yes & 3 & Pulmonary fibrosis & 89, III & Respiratory failure \\
\hline 63 & M & COPD, CHF, IHD & $\begin{array}{l}\text { Yes, CHF, Staphylococcus aureus } \\
\text { from bronchial washings }\end{array}$ & 17 & Carcinomatosis & 83 , III & Respiratory failure \\
\hline
\end{tabular}

F: female; M: male; COPD: chronic obstructive pulmonary disease; RA: rheumatoid arthritis; MI: myocardial infarction; CHF: congestive heart failure; IHD: ischaemic heart disease. 
mortality and hence a review is necessary to ensure that quality of care is not an issue in the death of these patients. It is practical since the number of deaths is low enough to permit detailed review. The present study did indeed confirm the utility of this approach. There were 11 deaths among the 608 patients in risk classes I-III. There was one avoidable death in the study (the patient with prosthetic aortic valve stenosis and unilateral pulmonary oedema misdiagnosed as pneumonia), an indication that in practice patients in classes I-III who die must be reviewed, since one-third will not have pneumonia and most of the rest have comorbid disease of such severity as to result in death. This study has highlighted yet another limitation of the PSI score. Chronic obstructive pulmonary disease and interstitial pulmonary fibrosis are not included in the PSI scoring system, resulting in misclassification of the severity of illness of these patients [4]. If points were given for these two conditions, seven of the 11 patients would be in class IV.

MORTENSEN et al. [8] classified 208 deaths in a cohort of patients (from the PORT study) with CAP using the WHO protocol. Respiratory failure was the most common immediate cause of death accounting for $38 \%$ of all deaths. Pneumonia accounted for $4 \%$, and in $20 \%$ the immediate cause of death was unknown. The underlying cause of death was chronic lung disease in $10 \%$, pneumonia in $10 \%$, cardiac causes in $14 \%$, cancer in $24 \%$, neurological conditions in $29 \%$, and other conditions in $12 \%$. In the present study, chronic obstructive lung disease was the underlying cause of death in $18 \%$, pulmonary fibrosis in $27 \%$ and cancer in $27 \%$. Respiratory failure was the immediate cause of death in $64 \%$, cardiac causes $27 \%$, and pneumonia $9 \%$. The number of patients in this study is too small to conclude that there is a shift in the immediate and underlying causes of death in younger patients with CAP.

One of the limitations of this study is that all patients in risk classes I-III were classified as low risk for mortality. In the original study, patients in classes I and II were classified as low risk and patients in class III as intermediate risk [4]. However, this designation was given so that the PSI score could be used as a tool to guide the admission decision, in that patients in risk classes I and II could be managed at home and patients in class III would require a period of observation of a few hours before a decision as to the site of care was made [4]. Subsequent studies have shown that there may be medical and psychosocial contraindications to outpatient care among patients classified as low risk; some patients with conditions (e.g. immunosuppression, pulmonary fibrosis) that are important in the decision to admit are not included in the model's predictors. The PSI score was validated as a mortality prediction rule and not as a method for triage of patients with CAP [11].

The PSI score was developed for patients with a proven diagnosis of pneumonia. In practice misdiagnosis of pneumonia is common. Such was the case in this study in that three of the 11 patients were subsequently shown not to have pneumonia and another patient probably did not have pneumonia. Thus, guidelines for the management of CAP need to pay more attention to conditions that masquerade as pneumonia.

In summary, this study shows that a review of deaths in patients with community-acquired pneumonia who fall into risk classes I-III is a useful quality assurance tool. Some of these patients were misdiagnosed as pneumonia, and the absence of points for chronic obstructive pulmonary disease and interstitial lung disease resulted in a lower risk classification for some patients than was indicated by the severity of their illness. In addition, one potentially preventable death was identified.

\footnotetext{
Acknowledgements. The author would like to thank the following community-acquired pneumonia pathway nurses: J. de Jager, L. Gardner, L. Korobanik, T. Pfeiffer, C. Proskow, S. Marshall, N. Baker, N. Horne and F. Herbert.
}

\section{References}

1. Dixon RE. Economic costs of respiratory tract infections in the United States. Am J Med 1985; 78: 45-51.

2. National Centre for Health Statistics. National hospital discharge survey: annual summary 1990. Vital Health Stat 1992; 13: 1-225.

3. Marrie TJ, Lau CY, Wheeler SL, et al. A controlled trial of a critical pathway for treatment of community-acquired pneumonia. JAMA 2000; 283: 749-755.

4. Fine MJ, Auble TE, Yealy DM, et al. A prediction rule to identify low-risk patients with community-acquired pneumonia. N Engl J Med 1997; 336: 243-250.

5. Statistics Canada. 1996 Census of Canada. Toronto, ON, 1996.

6. Marrie TJ, Michalyk D, Mann P, and the CommunityAcquired Pneumonia Critical Pathway Team. A critical pathway for treating community-acquired pneumonia. Can J CME 2001; 13: 43-57.

7. World Health Organization. Manual of the International Classification of Diseases, Injuries, and Causes of Death. Geneva, World Health Organization, 1977.

8. Mortensen EM, Coley CM, Singer DE, et al. Causes of death for patients with community-acquired pneumonia. Results from the pneumonia patient outcomes research team cohort study. Arch Intern Med 2002; 162: 1059-1064.

9. Rhew DC, Goetz MB, Shekelle PG. Evaluating quality indicators for patients with community-acquired pneumonia. Jt Comm J Qual Improv 2001; 27: 575-590.

10. Marrie TJ, Carriere KC, Yin J, Johnston DH. Mortality during hospitalization for pneumonia in Alberta, Canada is associated with physician volume. Eur Respir J 2003; 22: 148-155.

11. American College Emergency Physicians. Clinical policy for the management and risk stratification of communityacquired pneumonia in adults in the emergency department Ann Emerg Med 2001; 38: 107-113. 